\title{
Evaluación experimental de la toxicidad de cobre in situ sobre la fauna asociada a Perumytilus purpuratus (Bivalvia: Mytilidae), un ingeniero ecosistémico
}

Experimental evaluation of the in situ copper toxicity on associated fauna of Perumytilus purpuratus (Bivalvia: Mytilidae), an ecosystem bioengineer

\author{
Jesús Acevedo ${ }^{1}$, Fernanda I. Orellana ${ }^{1}$ y Ricardo Guiñez ${ }^{1}$
}

\author{
${ }^{1}$ Instituto de Investigaciones Oceanológicas, Facultad de Recursos del Mar, Universidad de Antofagasta, Casilla 170, \\ Antofagasta, Chile.rguinez@uantof.cl
}

\begin{abstract}
In this study we evaluated the effects of copper pollution on the mussel Perumytilus purpuratus-associated macrofaunal community on the basis of a pilot experiment in situ (northern Chile), using a modificated technique of copper administration. We used three copper treatments (Control, PRESS-continuous copper administration, and PULSE-intermittently copper administration) applied on three mussel matrices or beds. The results evidenced the presence of 46 taxa associated to the mussel P. purpuratus. Significant effects of the treatments of copper on mussel density, maximum length and number of strata, and the faunal species richness and equitability were not detected. However, marginally significant effects of the treatments of copper on the diversity index were observed. The analysis of the faunistic composition using a non metric multidimensional scaling showed a significant effect of the PRESS and PULSE treatments, producing a divergent behavior regarding the Control. This suggests that the P. purpuratus-associated fauna respond differentially to copper frequency administration and we suggest that it is possible to use the associated fauna as a sentinel or community bio-indicator for the tracking of the effects of copper and other heavy metals.
\end{abstract}

Key words: Copper pollution, mussel beds, bivalve

Resumen. - En este estudio se evaluó la influencia que tiene la contaminación por cobre sobre la macrofauna asociada a las matrices de P. purpuratus sobre la base de un experimento piloto in situ (norte de Chile), de pequeña escala aplicando una técnica modificada de administración de cobre. El experimento se implementó usando un diseño experimental bifactorial de bloques aleatorios, con tres tratamientos de suministro de cobre, (Control, Continuo (PRESS) y por Pulsos (PULSE)). Los resultados mostraron la presencia de una fauna de 46 taxa asociados a la matrices de P. purpuratus. No se detectó efectos significativos de los tratamientos de cobre sobre la densidad, longitud máxima y número de estratos de los mitílidos, ni de la riqueza de especies (S) y la uniformidad (J ). Sin embargo, se observó efectos marginalmente significativos de los tratamientos de cobre sobre el índice de diversidad. El análisis de la composición faunística mediante escalamiento multidimensional métrico mostró diferencias significativas entre los tratamientoS PRESS y PULSE, con un comportamiento divergente con respecto al control. Esto permite demostrar que el cobre y su modo de administración puede producir cambios observables en la macrofauna asociada a P. purpuratus, lo que permite proponer la utilización de la fauna asociada a este ingeniero ecosistémico, como un centinela o un bioindicador comunitario para el seguimiento de los efectos del cobre y de otros metales pesados.

Palabras clave: Contaminación por cobre, matrices del chorito, bivalvo

\section{INTRODUCCIÓN}

Desde los trabajos tempranos de Goldberg (1975) y Phillips (1976), los moluscos marinos han sido ampliamente usados como bioindicadores de contaminación. En particular, las especies de mitílidos presentan características que los hacen particularmente convenientes para estudiar el impacto de los contaminantes sobre los ecosistemas marinos, debido a sus amplias distribuciones geográficas, su adultez sésil y su hábitat costero intermareal, donde generalmente se producen problemas de contaminación (Viarengo \& Canesi 1991, Regoli \& Principato 1995, Boening 1999). Adicionalmente, algunos mitílidos son importantes ingenieros ecosistémicos en los sistemas marinos bentónicos debido a que se agregan en matrices y modifican la naturaleza y complejidad del sustrato (Alvarado \& Castilla 1996, Guiñez \& Castilla 1999, Borthagaray \& Carranza 2007). Algunos grupos de 
organismos pueden ser favorecidos por la complejidad estructural o la heterogeneidad ambiental de las matrices de mitílidos (Prado 2004, Prado \& Castilla 2006, Valdivia \& Thiel 2006, Borthagaray \& Carranza 2007). Sin embargo, no disponemos de ninguna evidencia sobre el potencial efecto de la contaminación sobre la fauna asociada a estos ingenieros ecosistémicos, en particular, de tóxicos químicos tales como metales pesados, que representan la perturbación antropogénica más prevalente dentro de los ambientes marinos del mundo (Piola \& Johnston 2008).

El cobre es un tóxico común en los ambientes marinos (Viarengo et al. 1994, Piola \& Johnston 2006), a pesar de que en concentraciones trazas es un metal esencial presente en los organismos vivos e involucrado en sus procesos metabólicos claves, tales como el transporte de electrones en la fotosíntesis y en la operación de varios sistemas enzimáticos (Riveros 2002, Riveros et al. 2002, Rodríguez de la Rua et al. 2002, Riveros et al. 2003, Acosta \& Lodeiros 2004).

En la zona norte de Chile, con un gran desarrollo de la minería del cobre, se ha sugerido e identificado la existencia de ecosistemas costeros impactados negativamente por la presencia de altas concentraciones de este metal producto de los relaves mineros, como por ejemplo los casos de Puerto de Chañaral y Taltal (Lee \& Correa 2004, 2005, Ramírez et al. 2005, Stauber et al. 2005, Lee \& Correa 2007), o en puertos de carga y descarga del mineral, como por ejemplo el Puerto de Antofagasta (Bahía San Jorge) (Riveros 2002, Riveros et al. 2002, 2003, Salamanca et al. 2004). Adicionalmente, la contaminación por metales puede también ocurrir en la forma de pinturas 'antifouling', residuos y descargas domésticas, industriales y mineras, corrosión de cañerías y basuras metabólicas (Salamanca et al. 2004, Piola \& Johnston 2008).

Mucho de nuestro conocimiento actual de los efectos antrópicos de la contaminación sobre los ambientes naturales procede de pruebas de toxicidad realizadas en condiciones convencionales de laboratorio siendo necesario realizar pruebas toxicológicas en condiciones naturales in situ, de modo tal que permitan superar las restricciones que presentan las pruebas toxicológicas de laboratorio (Johnston et al. 2002). Experimentos en condiciones naturales, permitirían - entre otros aspectos - simular eventos más realistas de contaminación de distintas escalas espaciales y temporales. En consecuencia, uno de los principales desafíos para los ecotoxicólogos es la necesidad de incrementar la relevancia de las pruebas de toxicidad para predecir acertadamente los efectos de la liberación de tóxicos en los ambientes naturales. En este sentido los ecotoxicólogos han identificado tres tareas relevantes: 1) usar experimentación de campo para poder validar las predicciones de los estudios de laboratorio, 2) realizar pruebas de toxicidad en niveles de organización superiores a la de individuos con el propósito de detectar efectos directos e indirectos en las especies y 3 ) tomar en cuenta los efectos que produce la variación de la exposición a los contaminantes; como por ejemplo, si la exposición es prolongada o intermitente. A pesar de que existen respuestas diferenciales de las especies a los tóxicos entre experimentos de laboratorios y de campo, siguen siendo raros los estudios experimentales en donde se haya medido la respuesta a los tóxicos en condiciones de campo (Johnston \& Webb 2000, Lindegarth \& Underwood 2002, Johnston \& Keough 2003).

De este modo, mediante el suministro de un metal potencialmente tóxico, tal como el cobre, el cual puede ser administrado en forma constante o alternada en el tiempo, se podría determinar experimentalmente si sus efectos sobre la fauna asociada a ingenieros ecosistémicos son diferenciales. Esto también podría permitir comprender de modo más preciso cómo estos tóxicos y sus regímenes de impacto pueden afectar a las comunidades biológicas, sus componentes específicos y sus interacciones. Lo anterior podría generar un mejor conocimiento experimental para fundamentar medidas de control o de políticas de manejo de eventos de contaminación más realistas (Johnston \& Keough 2003).

Como un aporte al estudio en condiciones de campo de los efectos de tóxicos, Johnston \& Webb (2000) propusieron dos técnicas novedosas para la evaluación de la toxicidad del cobre sobre ensambles de organismos incrustantes ('fouling'). A partir de estas técnicas se han realizado investigaciones en relación con ensambles de organismos incrustantes y su reclutamiento (Johnston \& Keough 2000, Johnston et al. 2002, Johnston \& Keough 2003). En el presente trabajo se propuso usar una de estas técnicas (Johnston \& Webb 2000) para evaluar por primera vez el efecto de la toxicidad del cobre sobre la fauna asociada a un mitílido en condiciones de campo. Con este propósito implementamos un experimento piloto de pequeña escala (decenas de metros) para explorar in situ los posibles efectos diferenciales del tipo de régimen de administración del cobre -constante o por pulso- sobre la composición faunística y biodiversidad de la macrofauna asociada a las matrices de Perumytilus purpuratus (Lamarck, 1819) y al mismo tiempo evaluar si existe un efecto sobre la matriz de mitílidos. 


\section{MATERIAL Y MÉTODOS}

\section{SITIO DE ESTUDIO}

Este trabajo se desarrolló entre el 5 de febrero y el 25 de mayo de 2007 en los roqueríos costeros del área sur de la ciudad de Antofagasta, sector denominado Asociación de Automovilistas de Antofagasta, dentro de la Bahía San Jorge, también conocida como Bahía de Antofagasta, norte de Chile $\left(23^{\circ} 42^{\prime} 18.85^{\prime \prime} \mathrm{S}, 70^{\circ} 25^{\prime} 39.59^{\prime \prime} \mathrm{W}\right)$. El experimento se implementó sobre plataformas planas (pendientes entre 0 y $10^{\circ}$ ) con $100 \%$ de cobertura de matrices de Perumytilus purpuratus en la franja media superior (Castilla 1981, Castilla et al. 2004a).

\section{Administración de COBRE a las MATRICES}

La administración de cobre a las matrices de mitílidos se realizó según Johnston \& Webb (2000) pero en vez de $\mathrm{CuSO}_{4}$ se utilizó $\mathrm{Cu}^{+2}$ líquido. Específicamente, se construyeron moldes cilíndricos de yeso piedra (dental) con una concentración de $15 \mu \mathrm{g} \mathrm{l}^{-1}$ de cobre, usando un tubo de PVC de $60 \mathrm{~mm}$ de largo por $32 \mathrm{~mm}$ de diámetro y adicionando $0,6 \mathrm{ml}$ de cobre líquido $\mathrm{Cu}^{+2}$ (Fluka Chemika) a una mezcla de $40 \mathrm{~g}$ de yeso y $20 \mathrm{ml}$ de agua de mar filtrada y esterilizada con UV, secando por $24 \mathrm{~h}$ a temperatura ambiente. Posteriormente la mitad superior del cilindro de PVC se cortó y retiró, cuidando de no dañar el molde interior de yeso (Fig. 1) y quedando expuesto en su mitad superior. Esta unidad de cilindro de yeso se instaló sobre un sistema de anclaje de PVC (tapa de PVC de 32 $\mathrm{mm}$ ) usando un perno de acero inoxidable con ayuda de un taladro de percusión a baterías (Bosch GSB 24 VE-2 Profesional) (Fig. 1). Esto permitió poner y sacar unidades de cilindros de yeso en el sistema de anclaje de modo rápido y expedito. El valor de $15 \mathrm{\mu g} \mathrm{l}^{-1}$ de cobre administrado se estableció con la finalidad de cumplir con la normativa chilena del Anteproyecto para el Establecimiento de las Normas Secundarias de Calidad Ambiental para Aguas Continentales Superficiales y Marinas ${ }^{1}$. Con la aplicación de esta técnica se busca explícitamente producir efectos locales que sean capaces de generar sucesos de contaminación transientes.

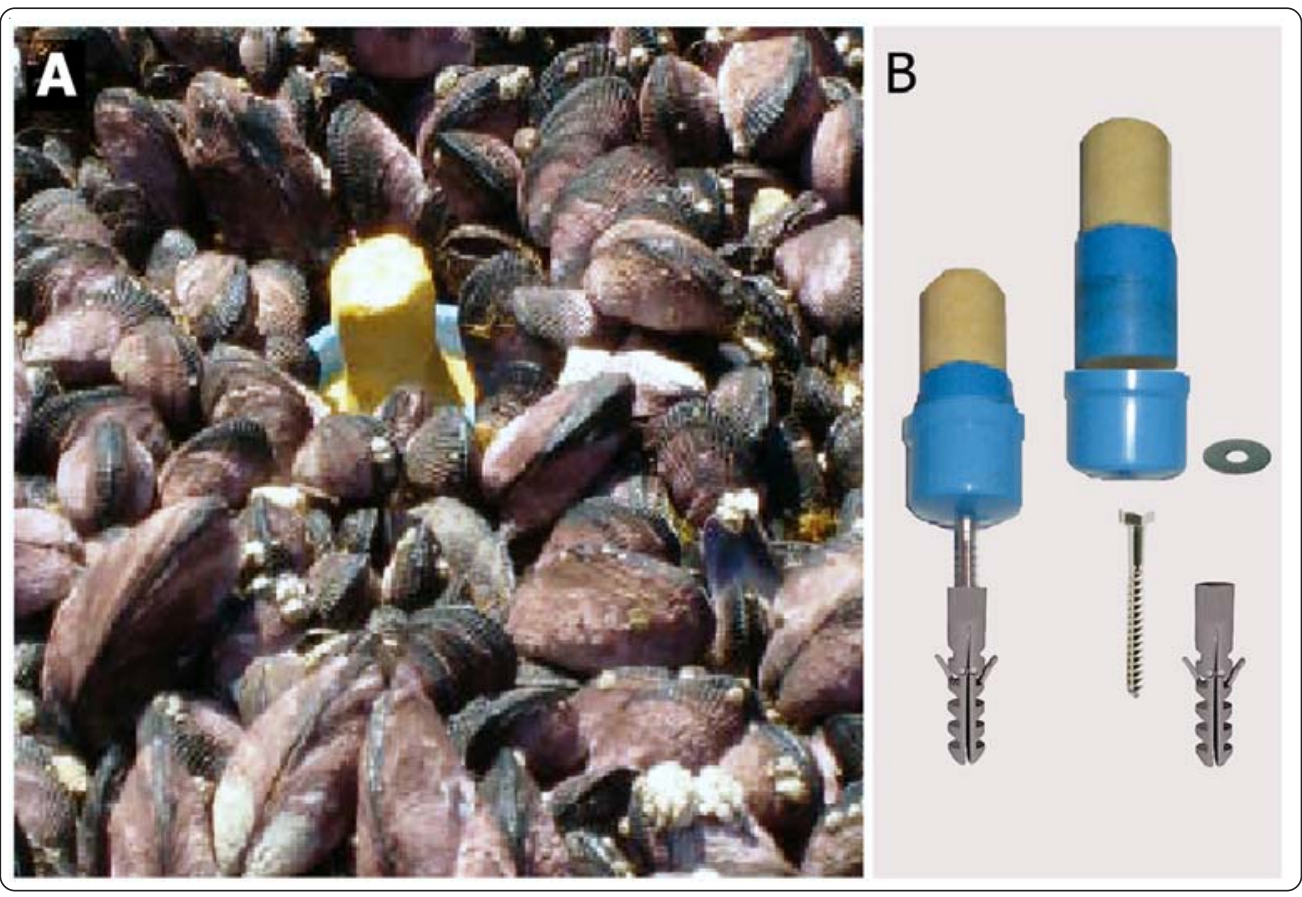

Figura 1. A) Dispositivo de administración de cobre inserto en unidad experimental en condiciones de terreno. B) Partes del dispositivo de administración, bloque de yeso y sistema de anclaje / A) Copper device of administration inserted in experimental unit at the field. B) Device of administration set, gypsum block and anchoring system

${ }^{1}$ Guía CONAMA para el establecimiento de las normas secundarias de calidad ambiental para aguas continentales superficiales y marinas. বhttp:// www.bcn.cl/ carpeta_temas/ temas_portada.2005-12-27.4449440028/ GuiaNormaPract.pdf> 


\section{DISEÑO EXPERIMENTAL}

Se implementó un diseño factorial mixto de dos vías (tratamiento de cobre considerado como factor fijo y matrices de $P$. purpuratus considerado como factor aleatorio) sin réplica. Se implementó cuatro tratamientos de cobre, que se aplicaron en cada una de tres matrices independientes seleccionadas al azar. La administración de cobre se efectuó de modo permanente (tratamiento PRESS: contaminación constante) y a través de pulsos (tratamiento PULSE: contaminación intermitente). Para el tratamiento PRESS las unidades de yeso con cobre fueron reemplazadas cada dos semanas durante todo el periodo experimental. En experimentos previos se determinó que las unidades de yeso tenían una duración de más de dos semanas. En cambio, en el caso PULSE se fue alternando cada dos semanas con y sin unidades de yeso con cobre. Adicionalmente, se consideraron otros dos tratamientos: i) un tratamiento control que consistió en ninguna manipulación a la unidad experimental en la matriz, ii) tratamiento control, denominado como blanco, que consistió en unidades de yeso sin cobre, el cual fue aplicado sólo de modo continuo.

Las tres matrices con $100 \%$ de cobertura usadas en este experimento estaban alejadas unas de otras a una distancia mínima de cinco metros, y sus dimensiones variaron entre 108,46 y $150 \mathrm{~m}^{2}$ de superficie. En cada una de estas matrices se asignó aleatoriamente los cuatro tratamientos de cobre, con el cuidado de que cada tratamiento estuviera alejado del otro por al menos un metro de distancia.

El experimentó tuvo una duración de 85 días. El área de muestreo correspondió a una circunferencia de 254,5 $\mathrm{cm}^{2}$ mayor al tamaño mínimo de muestra $\left(225 \mathrm{~cm}^{2}\right)$ determinada por Prado (2004) como representativo para la fauna asociada a las matrices de Perumytilus purpuratus (Fig. 1). Tres días antes del inicio del experimento se extrajo tres muestras en cada bloque (matriz) de $P$. purpuratus con la finalidad de tener un patrón de comparación inicial tanto de las características poblacionales de las matrices de mitílidos como de la diversidad de especies. Al terminar el experimento se obtuvo una muestra centrada en el punto donde se aplicó cada uno de los cuatro tratamientos. Todos los mitílidos fueron extraídos, separándolos cuidadosamente y cortando sus bisos, luego se retiró la fauna asociada mayor a 300 micrones, siguiendo a Prado \& Castilla (2006). No se consideró a los anfípodos e isópodos, puesto que se escapaban con facilidad al momento de la recolección. Los poliquetos fueron sólo determinados hasta el nivel de familia, con excepción de Nereis sp. Los sistemas de anclaje ocuparon un área despreciable $\left(8,04 \mathrm{~cm}^{2}\right)$, y no se corrigió por su efecto. Las muestras de mitílidos y la macrofauna asociada obtenida fueron conservadas en etanol alcohol 70\%. Cada mitílido fue medido en su longitud máxima, longitudes antero-posterior, latero-lateral y dorso-ventral usando un pie de metro electrónico. Se registró el número total de individuos y se obtuvo el peso húmedo total de los mitílidos y del sedimento retenido dentro de cada unidad experimental. El número de estratos de las muestras se obtuvo siguiendo a Guiñez \& Castilla (1999).

\section{ANÁlisis ESTAdístico de los DATOS}

La riqueza de especies se determinó como el número de especies en cada unidad experimental (área de muestreo), y la biodiversidad se estimó usando la riqueza de especies (S), el índice de diversidad de Shannon (H') y el de Uniformidad (J) usando el software PAST (Hammer et al. 2001). Por otra parte, se determinó la densidad de mitílidos, el número de estratos y el peso de sedimentos para cada unidad experimental. Para el análisis estadístico de estas variables se aplicó un análisis multivariado de varianzas (ANMUDEVA) y análisis de varianzas univariado (ANDEVA) de dos vías sin interacción, usando el software SAS 9.1, y considerando un diseño de bloques aleatorios con el modelo estadístico $\mathrm{y}_{\mathrm{ij}}=\mu+\mathrm{T}_{\mathrm{i}}+\mathrm{B}_{\mathrm{j}}+\mathrm{e}_{\mathrm{ij}}$, en donde $\mathrm{y}_{i j}$ es la variable respuesta, $\mu$ es la media poblacional, $\mathrm{T}$ es el efecto fijo de los $i$ tratamientos, $\mathrm{B}$ es el efecto aleatorio de las $j$ matrices consideradas como bloques y $e$ es el error residual de los $i$ tratamientos y $j$ bloques. Este diseño permite tanto controlar el efecto de las matrices de mitílidos, como también obtener una mejor estimación de los efectos de tratamientos de cobre. Para verificar las presunciones de normalidad (prueba de KolmogorovSmirnov) y de homogeneidad de varianzas (prueba de Bartlett) se utilizó el programa MINITAB 14.0. El ANMUDEVA se utilizó para estimar las diferencias del modelo sobre las tres variables respuestas ( $\mathrm{S}, \mathrm{H}^{\prime}$ y $\mathrm{J}$ ) simultáneamente. Para analizar las diferencias en la composición faunística de la comunidad asociada a Perumytilus purpuratus, se uso los nuevos métodos y software desarrollados por Anderson (2001). En particular, usamos ANMUDEVA no paramétrico (np-MANOVA) y PERMANOVA DISTL (que permite modelar la matriz de diseño apropiada para el modelo estadístico aplicado, ver arriba) usando el método de permutaciones, y aplicamos la estrategia de escalamiento métrico multidimensional a través del método de coordenadas principales ('principal coordinates', PCO). Para determinar las principales especies que determinaron las diferencias en la 
composición faunística se usó el algoritmo SIMPER (Clarke 1993).

\section{Resultados}

En total se encontraron 46 taxa asociados a las matrices de Perumytilus purpuratus, pertenecientes a 10 Phyla (Tabla 1). Los taxa más comunes correspondieron a: Annelida $(\mathrm{N}=14)$, Mollusca $(\mathrm{N}=11)$ y Arthropoda $(\mathrm{N}=$ 9) (Tabla 1). Las variables riqueza de especies, índice de diversidad y uniformidad, junto a la densidad de mitílidos, el número de estratos y el peso seco de sedimento cumplieron con las presunciones de normalidad (Kolmogorov-Smirnov; $P>0,150$ ) y de homogeneidad de varianzas (prueba de Bartlett; $P>0,116$ ). Tanto la longitud (promedio $=21,063 \mathrm{~mm} \pm 5,971$ D.E.), la densidad (promedio $=19.569$ ind $\mathrm{m}^{-2} \pm 5.485$ D. E.), el número de estratos (promedio $=3,26 \pm 1,01$ D.E.) como el peso seco total de sedimentos $(462,4 \mathrm{~g} \pm 274,3$ D.E. $)$ no mostraron diferencias significativas asignables al efecto de tratamientos (ANDEVA; $P>0,144$ ).

No se detectó diferencias significativas entre los tratamientos control y blanco (ANDEVA; $P>0,33$ ) para las variables riqueza de especies, índice de diversidad y uniformidad, por lo que estas variables fueron combinadas (pooled) y nominadas como control para los análisis posteriores.

El tratamiento PRESS mostró los menores valores de riqueza de especies y del índice de diversidad en relación con PULSE y CONTROL (Fig. 2), y la uniformidad no mostró tendencias claras en función de los tratamientos de cobre. El ANMUDEVA no detectó diferencias significativas para el efecto de tratamiento $(P=0,227)$, aunque si se observó un efecto marginal de la variable de bloqueo (matrices) $(P=0,059)$. Por otra parte, el ANDEVA univariado mostró que hubo un efecto marginalmente significativo de tratamiento sobre el índice de diversidad (Tabla 2B; $P=0,060)$. El análisis a priori de contrastes ortogonales permitió detectar diferencias significativas entre los tratamientos PULSE y PRESS (Tabla 2B; $P=$ 0,022) para el índice de diversidad, y también un efecto significativo de bloqueo sólo para la uniformidad (Tabla 2C; $P=0,008)$, lo que sugiere un efecto de las matrices sobre las varianzas de esta variable.

Tabla 1. Listado de macroinvertebrados asociados a los mantos de Perumytilus purpuratus correspondiente al total de unidades experimentales / Macroinvertebrates list associated to Perumytilus purpuratus matrices over all the data

\begin{tabular}{|c|c|c|}
\hline $\begin{array}{l}\text { ARTHROPODA (9) } \\
\text { Acanthocyclus gayi } \\
\text { Allopretrolisthes angulosus } \\
\text { Cyclograpsus cinereus } \\
\text { Petrolisthes gramulosus } \\
\text { Petrolisthes violaceus } \\
\text { Petrolisthes sp. } \\
\text { Jehlius cirratus } \\
\text { Balanus laevis } \\
\text { Notochthamalus scabrosus } \\
\text { MOLLUSCA (11) } \\
\text { Chaetopleura peruviana } \\
\text { Chiton granosus } \\
\text { Tonicia } \text { sp. } \\
\text { Concholepas concholepas } \\
\text { Gastropoda sp. } \\
\text { Nodilittorina peruviana } \\
\text { Scurria ceciliana } \\
\text { Scurria } \text { sp. } \\
\text { Lottia } \text { sp. } \\
\text { Turritella cingulata } \\
\text { Protothaca thaca }\end{array}$ & $\begin{array}{l}\text { ECHINODERMATA (4) } \\
\text { Heliasther helianthus } \\
\text { Holothuroidea sp. } \\
\text { Tetrapygus niger } \\
\text { Ophiuroidea sp. } \\
\text { CNIDARIA (4) } \\
\text { Phymactis papillosa } \\
\text { Phymanthea pluvia } \\
\text { Actinidae sp. } 1 \\
\text { Actinidae sp. } 2 \\
\text { ANNELLIDA (11) } \\
\text { Arabellidae sp. } 1 \\
\text { Cirratulidae sp. } 1 \\
\text { Flabelligeridae sp. } 1 \\
\text { Lumbrineridae sp. } 1 \\
\text { Nereidae sp. } 1 \\
\text { Nereidae sp. } 2 \\
\text { Nereis sp. } \\
\text { Orbiniidae sp. } 1 \\
\text { Polynoidae sp. } 1 \\
\text { Polynoidae sp. } 2 \\
\text { Sabellidae sp. } 1\end{array}$ & $\begin{array}{l}\text { CHORDATA (1) (ASCIDIACEA) } \\
\text { Pyura praeputialis } \\
\text { NEMERTEA (1) } \\
\text { Nemertino non det. } \\
\text { NEMATODA (2) } \\
\text { Nematoda sp. } 1 \\
\text { Nematoda sp. } 2 \\
\text { PLATHELMINTHES (2) } \\
\text { Turbellaria } \\
\text { Turbellaria ind. } \\
\text { SIPUNCULA (1) } \\
\text { Sipunculidae }\end{array}$ \\
\hline
\end{tabular}




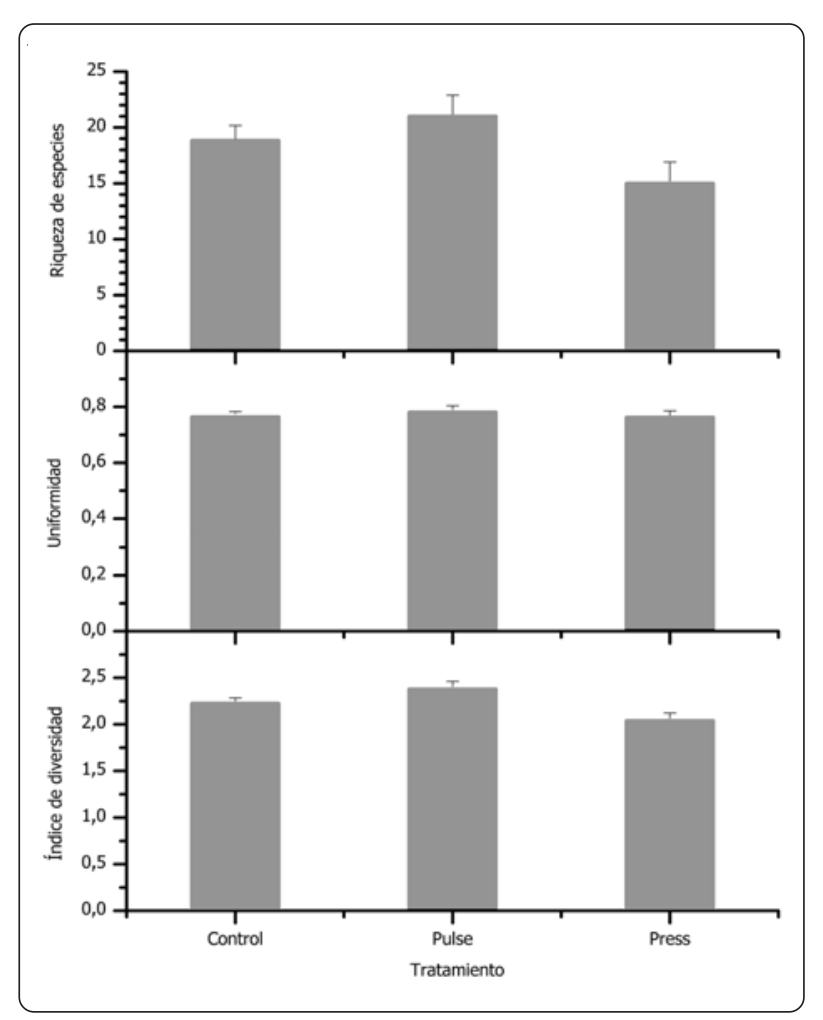

Figura 2. Promedio y error estándar (líneas sobre las barras) de la riqueza de especie, uniformidad e índice de diversidad, para cada tratamiento implementado (Control, Pulse y Press) / Mean and standard error (lines over bars) of the species richness, equitability and diversity index for each implemented treatment (Control, Pulse and Press)

La composición faunística no mostró diferencias significativas entre los tratamientos control y blanco (PERMANOVA DISTL; $P=0,137$ ) por lo que ambos tratamientos fueron combinados y denominados control. El análisis de escalamiento métrico multidimensional (MDS) de la composición faunística (Fig. 3) muestra, sobre la proyección de los dos primeros ejes de coordenadas, una segregación de los tratamientos, con los controles en posición central y los tratamientos PRESS y PULSE segregándose hacia extremos disjuntos. El efecto de los tratamientos sobre la composición faunística fue significativo (np-MANOVA y PERMANOVA DISTL; $P$ $=0,025 ;$ stress $=0,11)$, y explicado fundamentalmente por la diferencia estadísticamente significativa $(P=0,034)$ observado sólo entre los tratamientos PRESS y PULSE. Este comportamiento divergente entre PRESS y PULSE (Fig. 3) con una disimilaridad faunística del 50,71\%, está asociada con las respuestas diferenciales observadas para los taxa: Nereidae sp. 2, Cirratulidae, Actinidae sp. 1,
Tabla 2. Análisis de varianza mixto de dos vías (tratamiento de cobre y bloque) sin réplicas de las variables respuestas: riqueza de especies, Índice de diversidad y uniformidad / Two way mixed analysis of variance (copper treatment and block) without replicates of the dependent variables: species richness, diversity index and equitability

\begin{tabular}{|c|c|c|c|c|}
\hline & \multicolumn{4}{|c|}{ Fuente de Variación } \\
\hline & DF & MS & $\mathrm{F}$ & $P$ \\
\hline \multicolumn{5}{|l|}{ Riqueza de Especies } \\
\hline Tratamiento de Cobre & 2 & 28,0417 & 2,59 & 0,144 \\
\hline Control vs Pulse/Press & 1 & 2,0833 & 0,19 & 0,674 \\
\hline Control vs Pulse & 1 & 9,3889 & 0,87 & 0,382 \\
\hline Control $v s$ Press & 1 & 29,3889 & 2,72 & 0,143 \\
\hline Pulse vs Press & 1 & 54,0000 & 5,00 & 0,064 \\
\hline Bloque & 2 & 31,5833 & 2,92 & 0,119 \\
\hline Error & 7 & 10,8095 & & \\
\hline \multicolumn{5}{|l|}{ Índice de Diversidad } \\
\hline Tratamiento de Cobre & 2 & 0,0860 & 4,29 & 0,060 \\
\hline Control vs Pulse/Press & 1 & 0,0007 & 0,04 & 0,857 \\
\hline Control vs Pulse & 1 & 0,0472 & 2,35 & 0,169 \\
\hline Control vs Press & 1 & 0,0679 & 3,39 & 0,108 \\
\hline Pulse vs Press & 1 & 0,1714 & 8,54 & 0,022 \\
\hline Bloque & 2 & 0,0181 & 0,90 & 0,448 \\
\hline Error & 7 & 0,0201 & & \\
\hline \multicolumn{5}{|l|}{ Uniformidad } \\
\hline Tratamiento de Cobre & 2 & 0,0003 & 0,18 & 0,839 \\
\hline Control vs Pulse/Press & 1 & 0,0001 & 0,05 & 0,824 \\
\hline Control vs Pulse & 1 & 0,0004 & 0,26 & 0,627 \\
\hline Control vs Press & 1 & 0,0000 & 0,02 & 0,899 \\
\hline Pulse vs Press & 1 & 0,0005 & 0,31 & 0,597 \\
\hline Bloque & 2 & 0,0165 & 10,62 & 0,008 \\
\hline Error & 7 & 0,0015 & & \\
\hline
\end{tabular}

Acanthocyclus gayi, Cyclograpsus cinereus, Phymactis clematis, Polynoidae sp. 1, Chiton granosus y Nemertino no determinado.

\section{Discusión}

En nuestro trabajo experimental se determinó un total de 46 taxa asociados a las matrices de Perumytilus purpuratus en la franja intermareal media superior de la Bahía de Antofagasta $\left(23^{\circ} \mathrm{S}\right)$. Este valor fue superior al número de especies de la fauna acompañante de Perumytilus purpuratus encontrado por Thiel \& Ullrich (2002) para las localidades de Taltal $\left(\mathrm{n}=27 ; 25^{\circ} \mathrm{S}\right)$ e Iquique $(\mathrm{n}=31$; $20^{\circ} \mathrm{S}$ ), que puede ser explicado por un incremento efectivo del número de especies en la Bahía de Antofagasta, dadas sus características particulares (Castilla \& Camaño 2001, Piñones et al. 2007) o por el menor tamaño muestral (50 $\mathrm{cm}^{2}$ ) usado por Thiel \& Ullrich (2002). Sin embargo, nuestros valores fueron inferiores a las 92 especies encontradas en Chile central (Prado 2004, Prado \& Castilla 


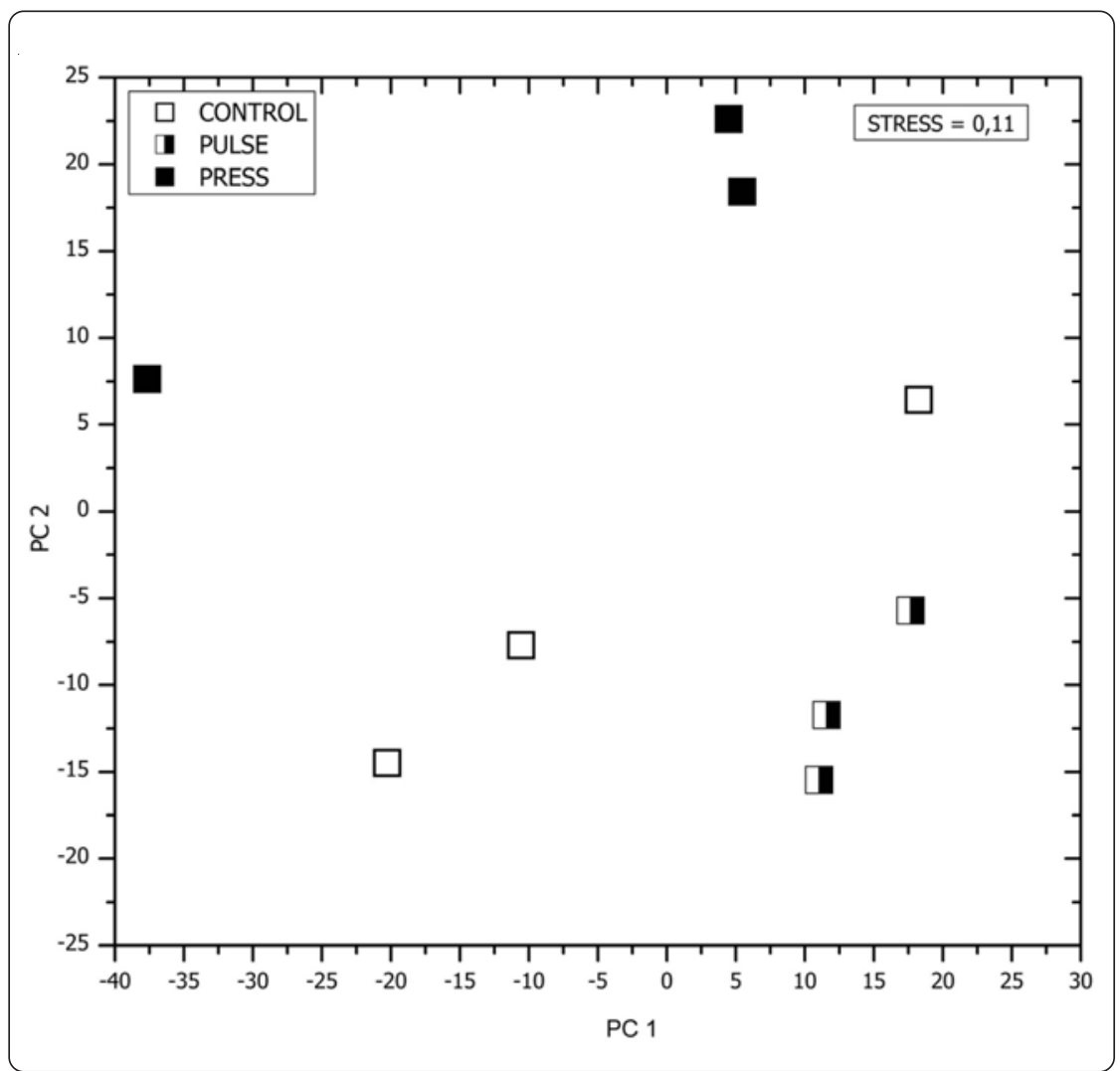

Figura 3. Análisis de escalamiento métrico multidimensional (MDS) para la fauna correspondiente a los distintos tratamientos (Control, Pulse y Press). PC corresponde a las principales coordenadas / Metric multidimensional scaling analysis (MDS) for the macrofauna associated to the different treatments (Control, Pulse and Press). PC correspond to the principal coordinates
2006) explicado quizás por diferencias latitudinales. Por otra parte, estos valores fueron mayores a las 27 especies determinadas para sitios rocosos sin especies dominantes tales como $P$. purpuratus y el tunicado Pyura praeputialis (Heller 1878) (Castilla et al. 2004a, b, Guiñez \& Castilla 2006). Este resultado sugiere que $P$. purpuratus también se estaría comportando como un bioingeniero autogénico en la Bahía de Antofagasta como fue propuesto en Chile central por Prado \& Castilla (2006). Por otra parte, nuestros valores fueron menores a los encontrados para la fauna asociada a las matrices $P$. praeputialis $(\mathrm{N}=79$ especies) (Cerda \& Castilla 2001, Castilla et al. 2004b, Guiñez \& Castilla 2006) en la franja media inferior del intermareal rocoso de Bahía de Antofagasta. Esto podría ser explicado por la diferencia de altura de mareas en el intermareal observado entre ambas especies (Castilla et al. 2004a).

En nuestro experimento no detectamos efectos significativos de tratamiento de cobre sobre las matrices de Perumytilus purpuratus, tanto para la densidad, el número de estratos como para la longitud máxima de los mitílidos. Pero por el contrario, se produjeron efectos significativos o marginalmente significativos de los tratamientos de cobre sobre la composición de la fauna asociada a las matrices de $P$. purpuratus y sobre el índice de diversidad. Esto sugiere que los mitílidos son menos sensitivos al cobre que su fauna asociada, esto corresponde a una evidencia documentada por primera vez en la literatura. Lo que permite sugerir que, en efecto, la fauna asociada puede ser un buen bioindicador centinela de los efectos de la contaminación por cobre. Por otra parte, es importante destacar también que la composición de la macrofauna puede ser un indicador del tipo de dosificación (PULSE, PRESS) en que el cobre este arribando a las áreas con matrices de mitílidos. La concentración usada en el experimento de $15 \mu \mathrm{g}^{-1}$ fue menor que la norma chilena $\left(50 \mu \mathrm{g} \mathrm{l}^{-1}\right)$ pero mayor que aquella encontrada para este sitio en rangos de concentraciones del agua de mar de 0,136 $\mu \mathrm{g}^{-1}$ a $1,96 \mathrm{\mu g}^{-1}$ (Rodríguez 1997, Riveros 2002, Salamanca et al. 2004).

Es interesante destacar que estos resultados corresponden a un experimento in situ y abierto, con matrices para las que se esperaría elevados recambios de 
especies de la fauna acompañante desde matrices vecinas. Por lo tanto, es sorprendente el efecto diferencial observado de la composición de especies entre la administración PRESS y PULSE de Cobre, en particular por el poco tiempo en que se aplicó el cobre (85 días), que contrasta por ejemplo con períodos de más de 15 semanas usados en otros estudios (e.g., Johnston \& Keough 2003, 2005). Por otra parte, la disminución del índice de diversidad encontrada asociada el tratamiento PRESS concuerda con la evidencia observacional y experimental obtenida por Lee \& Correa $(2005,2007)$ en la bahía de Chañaral $\left(25^{\circ} \mathrm{S}\right)$ norte de Chile, $322 \mathrm{~km}$ de costa lineal al sur de nuestro sitio de estudio. Estos autores describen una disminución de la diversidad de la meiofauna, en relación con los efectos permanentes y de gran escala de los relaves de cobre, lo que sería asignable al agua intersticial (porewater) asociada a los relaves, de acuerdo a la evidencia experimental.

Los resultados obtenidos que muestran respuestas significativas de la composición faunística en relación al modo de administración del cobre abren un novedoso modelo experimental a partir de nuestra propuesta metodológica de insertar cilindros de yeso con cobre en matrices de mitílidos usando efectos locales que son capaces de generar sucesos de contaminación transientes. También, nuestros resultados permiten sugerir que la fauna asociada a los mantos de Perumytilus purpuratus podría ser un buen centinela o bioindicador comunitario de la contaminación por cobre.

\section{Agradecimientos}

Se agradece a la colaboración en este estudio a Soledad Morales, Aruza Robles, Paula Ruz en la identificación de especies y a Vania Yel en el apoyo en terreno y mediciones. Este trabajo fue financiado gracias a los Proyectos MEL/ UA 4577 y FONDECYT \# 1050848 a RG.

\section{LITERATURA CITADA}

Acosta V \& C Lodeiros. 2004. Heavy metals in the clam Tivela mactroides Born, 1778 (Bivalvia: Veneridae) from coastal localities with different degrees of contamination in Venezuela. Ciencias Marinas 30: 323-333.

Alvarado JL \& JC Castilla. 1996. Tridimensional matrices of Perumytilus purpuratus on intertidal platforms with varying wave forces in central Chile. Marine Ecology Progress Series 133: 135-141.

Anderson M. 2001. A new method for non-parametric multivariate analysis of variance. Austral Ecology 26: 32-46.

Boening DW. 1999. An evaluation of bivalves as biomonitors of heavy metals pollution in marine waters. Environmental Monitoring and Assessment 55: 459-470.
Borthagaray AI \& A Carranza. 2007. Mussels as ecosystem engineers: Their contribution to species richness in a rocky littoral community. Acta Oecologica-International Journal of Ecology 31: 243-250.

Castilla JC. 1981. Perspectivas de investigación en estructura y dinámica de comunidades intermareales rocosas de Chile central. II. Depredadores de alto nivel trófico. Medio Ambiente, Chile 5: 190-215.

Castilla JC \& A Camaño. 2001. El Piure de Antofagasta, Pyura praeputialis (Heller, 1878): un competidor dominante e ingeniero de ecosistemas. En: Alveal K \& A Antezana (eds). Sustentabilidad de la biodiversidad, pp. 719-729. Editorial Universidad de Concepción, Concepción.

Castilla JC, R Guiñez, AU Caro \& V Ortiz. 2004a. Invasion of a rocky intertidal shore by the tunicate Pyura praeputialis in the Bay of Antofagasta, Chile. Proceedings of the National Academy of Sciences of the United States of America 101: 8517-8524.

Castilla JC, NA Lagos \& M Cerda. 2004b. Marine ecosystem engineering by the alien ascidian Pyura praeputialis on a mid-intertidal rocky shore. Marine Ecology Progress Series 268: 119-130.

Cerda M \& JC Castilla. 2001. Diversity and biomass of macro-invertebrates in intertidal matrices of the tunicate Pyura praeputialis (Heller, 1878) in the Bay of Antofagasta, Chile. Revista Chilena de Historia Natural 74: 841-853.

Clarke K. 1993. Non-parametric multivariate analyses of changes in community structure. Australian Journal of Ecology 18: 117-143.

Goldberg ED. 1975. The mussel watch - A first step in global marine monitoring. Marine Pollution Bulletin 6: 111-114.

Guiñez R \& JC Castilla. 1999. A tridimensional self-thinning model for multilayered intertidal mussels. American Naturalist 154: 341-357.

Guiñez R \& JC Castilla. 2006. Estudios de la biodiversidad asociada a una especie única de tunicado marino en la bahía de Antofagasta. En: Camaño A, JC Castilla \& JA Simonetti (eds). Minería y biodiversidad, pp. 45-55. Sociedad Nacional de Minería, Santiago.

Hammer Ø, DAT Harper \& PD Ryan. 2001. Past: Paleontological Statistics Software Package for Education and Data Analysis. Palaeontologia Electronica 4: 1-9.

Johnston EL \& MJ Keough. 2000. Field assessment of effects of timing and frequency of copper pulses on settlement of sessile marine invertebrates. Marine Biology 137: 10171029.

Johnston EL \& IA Webb. 2000. Novel techniques for field assessment of copper toxicity on fouling assemblages. Biofouling 15: 165-173.

Johnston EL \& MJ Keough. 2003. Competition modifies the response of organisms to toxic disturbance. Marine Ecology Progress Series 251: 15-26.

Johnston EL, MJ Keough \& PY Qian. 2002. Maintenance of species dominance through pulse disturbances to a sessile marine invertebrate assemblage in Port Shelter, Hong Kong. Marine Ecology Progress Series 226: 103-114. 
Lee MR \& JA Correa. 2004. Copper mine tailings disposal: consequences for the interstitial polychaete Saccocirrus sonomacus (Canalipalpata, Protodrilida). Journal of the Marine Biological Association of the United Kingdom 84: 603-606.

Lee MR \& JA Correa. 2005. The effects of copper mine tailings disposal on littoral meiofaunal assemblages in the Atacama region of northern Chile. Marine Environmental Research 59: 1-18.

Lee MR \& JA Correa. 2007. An assessment of the impact of copper mine tailings disposal on meiofaunal assemblages using microcosm bioassays. Marine Environmental Research 64: 1-20.

Lindegarth M \& AJ Underwood. 2002. A manipulative experiment to evaluate predicted changes in intertidal, macro-faunal assemblages after contamination by heavy metals. Journal of Experimental Marine Biology and Ecology 274: 41-64.

Phillips DJH. 1976. The common mussel Mytilus edulis as an indicator of pollution by zinc, cadmium, lead and copper: Effects of environmental variables on uptake of metals. Marine Biology 38: 59-69.

Piñones A, JC Castilla, R Guinez \& JL Largier. 2007. Nearshore surface temperatures in Antofagasta Bay (Chile) and adjacent upwelling centers. Ciencias Marinas 33: 37 48.

Piola RF \& EL Johnston. 2006. Differential resistance to extended copper exposure in four introduced bryozoans. Marine Ecology Progress Series 311: 103-114.

Piola RF \& EL Johnston. 2008. Pollution reduces native diversity and increases invader dominance in marine hardsubstrate communities. Diversity and Distributions 14: 329-342.

Prado L. 2004. Diversidad de invertebrados marinos asociada al chorito maico, Perumytilus purpuratus (Lamarck, 1819) en Chile central. Tesis de Biología Marina, Facultad de Ciencias del Mar, Universidad de Valparaíso, Valparaíso, $76 \mathrm{pp}$.

Prado L \& JC Castilla. 2006. The bioengineer Perumytilus purpuratus (Mollusca: Bivalvia) in central Chile: biodiversity, structural complexity and heterogeneity. Journal of the Marine Biological Association of the United Kingdom 86: 417-421.

Ramirez M, S Massolo, R Frache \& JA Correa. 2005. Metal speciation and environmental impact on sandy beaches due to El Salvador copper mine, Chile. Marine Pollution Bulletin 50: 62-72.

Regoli F \& G Principato. 1995. Glutathione, Glutathionedependent and antioxidant enzymes in mussel, Mytilus galloprovincialis, exposed to metals under field and laboratory conditions: implications for the use of biochemical biomarkers. Aquatic Toxicology 31: 143-164.
Riveros A. 2002. Estudio de la calidad ambiental de zonas intermareales en la Bahía San Jorge (II Región-Chile), mediante la evaluación de respuestas biológicas del mitílido Perumytilus purpuratus. Tesis de Doctorado en Ciencias Ambientales, Facultad de Ciencias Naturales y Oceanográficas, Universidad de Concepción, Concepción, $126 \mathrm{pp}$.

Riveros A, M Zuñiga \& A Larraín. 2003. Copper metallothionein-like proteins as exposure biomarker in native and transplanted intertidal populations of the mussel Perumytilus purpuratus from San Jorge Bay, Antofagasta, Chile. Bulletin of Environmental Contamination and Toxicology 70: 233-241.

Riveros A, M Zuñiga, A Hernández \& A Camaño. 2002. Cellular biomarkers in native and transplanted populations of the mussel Perumytilus purpuratus in the intertidal zones of San Jorge Bay, Antofagasta, Chile. Archives of Environmental Contamination and Toxicology 42: 303-312.

Rodríguez T. 1997. Estudio preliminar del contenido de metales pesados $(\mathrm{Cu}, \mathrm{Pb}, \mathrm{Zn}$ y $\mathrm{Hg})$ en agua de mar en Perumytilus purpuratus, en la Bahía San Jorge, Antofagasta. Seminario de Ingeniero en Acuicultura y Licenciado en Ciencias del Mar, Universidad de Antofagasta, Antofagasta, $82 \mathrm{pp}$.

Rodríguez de la Rua A, JM Arellano, ML Gonzáles de Canales, J Blasco \& C Sarasquete. 2002. Acumulación de cobre y alteraciones histopatológicas en el ostión Crassotrea angulata. Ciencias Marinas 31: 455-466.

Salamanca M, B Jara \& T Rodríguez. 2004. Niveles de Cu, $\mathrm{Pb}$ y $\mathrm{Zn}$ en aguas y Perumytilus purpuratus en Bahía San Jorge, norte de Chile. Gayana 68: 53-62.

Stauber J, S Andrade, M Ramirez, M Adams \& JA Correa. 2005. Copper bioavailability in a coastal environment of northern Chile: comparison of bioassay and analytical speciation approaches. Marine Pollution Bulletin 50: 1363 1372.

Thiel M \& N Ullrich. 2002. Hard rock versus soft bottom: the fauna associated with intertidal mussel beds on hard bottoms along the coast of Chile, and considerations on the functional role of mussel beds. Helgoland Marine Research 56: 21-30.

Valdivia N \& M Thiel. 2006. Effects of point-source nutrient addition and mussel removal on epibiotic assemblages in Perumytilus purpuratus beds. Journal of Sea Research 56: 271-283.

Viarengo A \& L Canesi. 1991. Mussels as biological indicators of pollution. Aquaculture 94: 225-243.

Viarengo A, N Arena, L Canesi, FAAlia \& M Orunesu. 1994. Structural and biochemical alteration in the gills of copperexposed mussels. En: Renzoni A, N Mattei, L Lari \& MC Fossi (eds). Contaminants in the environment. A multidisciplinary assessment of risks to man and other organisms, pp. 135-143, Lewis Publishers.

Recibido el 1 de julio de 2010 y aceptado el 21 de septiembre de 2010 